\title{
Asymptotically Abelian Systems
}

\author{
S. Doplicher*, R. V. Kadison**, D. KastleR**** \\ and Derek W. Robinson* \\ University of Aix-Marseille \\ France
}

Received May 15, 1967

\begin{abstract}
We study pairs $\{\mathfrak{A}, \alpha\}$ for which $\mathfrak{Q}$ is a $C^{*}$-algebra and $\alpha$ is a homomorphism of a locally compact, non-compact group $G$ into the group of *automorphisms of $\mathfrak{A}$. We examine, especially, those systems $\{\mathfrak{A}, \alpha\}$ which are (weakly) asymptotically abelian with respect to their invariant states (i.e. $\langle\Phi| A \alpha_{g}(B)$ $\left.-\alpha_{g}(B) A\right\rangle \rightarrow 0$ as $g \rightarrow \infty$ for those states $\Phi$ such that $\Phi\left(\alpha_{g}(A)\right)=\Phi(A)$ for all $g$ in $G$ and $A$ in $\mathfrak{A}$ ). For concrete systems (those with $\mathfrak{A}$ acting on a Hilbert space and $g \rightarrow \alpha_{g}$ implemented by a unitary representation $g \rightarrow U_{g}$ on this space) we prove, among other results, that the operators commuting with $\mathfrak{A}$ and $\left\{U_{g}\right\}$ form a commuting family when there is a vector cyclic under $\mathfrak{A}$ and invariant under $\left\{U_{g}\right\}$. We characterize the extremal invariant states, in this case, in terms of "weak clustering" properties and also in terms of "factor" and "irreducibility" properties of $\left\{\mathfrak{A}, U_{g}\right\}$. Specializing to amenable groups, we describe "operator means" arising from invariant group means; and we study systems which are "asymptotically abelian in mean". Our interest in these structures resides in their appearance in the "infinite system" approach to quantum statistical mechanics.
\end{abstract}

\section{Introduction}

In the general frame of quantum mechanics the physical observables are described as self-adjoint operators on a Hilbert space $\mathscr{H}$ and the bounded observables (corresponding to bounded operators) therefore generate a $C^{*}$-algebra acting on $\mathscr{H}$. The algebraic approach to field theory $[1,2]$ proposes to consider as physical only the local observables i.e. those corresponding to measurements performed within finite regions of space during a finite time. These observables are described mathematically as the self-adjoint elements of an incomplete $C^{*}$-algebra whose completion $\mathfrak{A}$, called the quasi-local algebra, is considered as the main

* Permanent address: Istituto di Fisica G. Marconi, Piazzale delle Science 5, Roma.

** Permanent address: Department of Mathematics, University of Pennsylvania Philadelphia, Penn. U.S.A.

*** Present address: Institut des Hautes Etudes Scientifiques, 91 - Bures sur Yvette, France.

* Present address: Division Théorique CERN, Genève 23. 
mathematical entity whose abstract algebraic structure contains the whole physical information (the choice of a particular faithful representation being a matter of arbitrariness without physical implications). Field theory is thus viewed mathematically as the investigation of the quasilocal algebra $\mathfrak{A}$ which is supposed to satisfy appropriate axioms of physical origin (locality, Lorentz invariance etc.). Whilst the physical (local) observables are described as elements of $\mathfrak{A}$, the physical states are represented as the normalized positive linear forms over $\mathfrak{A}$ (states over $\mathfrak{A}$ ). In this framework the invariance groups of the physical theory (group of space and time translations, Lorentz transformations etc.) are homomorphically mapped into the automorphism group of the $C *$-algebra $\mathfrak{A}$. The automorphism $\alpha_{g}$ corresponding to the group element $g$ describes physically the "shifting by $g$ " of the local observables.

One of the first problems arising in this approach is the investigation of the translationally invariant states over the algebra $\mathfrak{A}$, those being natural candidates for the description of the equilibrium states of statistical mechanics (more generally the states invariant under any group of physical transformations are of interest) $[3,4,4 \mathrm{a}]$.

In this connection recent studies have revealed that a number of general results depend upon only a very weak locality assumption about the quasi-local algebra (entailing only a small part of the structure required by physics) namely a property of asymptotic abelianness (cf. Definitions 1 and 2 below) $[5,6,7,8,9,10]$. It seems therefore interesting to investigate mathematically the systems $\{\mathfrak{U}, \alpha\}$ of a $C^{*}$-algebra $\mathfrak{A}$ and a homomorphic mapping $\alpha$ of a group $G$ into the automorphism group of $\mathfrak{A}$ which possess such an asymptotic abelian property. This is the purpose of the present paper.

Section $I$ is concerned with general groups $G$ and an abstract notion of mean of elements of $\mathfrak{A}$. Section II describes the case of an amenable group possessing invariant means and discusses the connection between the above notion and the invariant means over $G$.

Owing to the delay between the conclusion of this research and the writing of this paper (due to geographical dispersion of the authors) some results overlapping with ours meanwhile appeared in the literature $[9,9 \mathrm{a}]$.

\section{General Case}

Theorem 1. Let $g \rightarrow U_{g}$ be a unitary representation of the group $G$ on the Hilbert space $\mathscr{H}, \mathfrak{A}$ a $C^{*}$-algebra acting on $\mathscr{H}$ such that $\alpha_{g}(A)=U_{g} A U_{g}^{-1}$ is in $\mathfrak{A}$ for each $g$ in $G$. We denote furthermore by $E_{0}$ the orthogonal projection in $\mathscr{H}$ on the subspace $\left\{\psi \in \mathscr{H} \mid U_{g} \psi=\psi\right.$ for all $\left.g \in G\right\} ;$ by $\mathscr{R}$ the von Neumann algebra generated by $\mathfrak{A}$ and $\left\{U_{g} \mid g \in G\right\}$; by $P$ the central carrier of 
$E_{0}$ in $\mathscr{R}$; and we assume that, for all $p \in E_{0} \mathscr{H}$ and $A, B \in \mathfrak{A}$

Then

$$
\operatorname{Inf}_{g \in G^{\prime}}\left|\left(\varphi\left|A \cdot \alpha_{g}(B)-\alpha_{g}(B) \cdot A\right| \varphi\right)\right|=0 \text {. }
$$

(i) $E_{0} \mathscr{R} E_{0}$ is abelian ${ }^{1}$ so that $E_{0}$ is an abelian projection in $\mathscr{R}$ and $\mathscr{R} P$ is of type $I$ with its center isomorphic to $E_{0} \mathscr{R} E_{0}$.

(ii) If $\mathscr{R} E_{0} \mathscr{H}$ is dense in $\mathscr{H}$ (or equivalently, $\mathfrak{A} E_{0} \mathscr{H}$ is dense in $\mathscr{H}$; or if $P=I$; or if $T \in \mathscr{R}^{\prime} \rightarrow T E_{0} \in \mathscr{R}^{\prime} E_{0}$ is an isomorphism between von Neumann algebras) then $\mathscr{R}$ is of type $I$ with its center isomorphic to $E_{0} \mathscr{R} E_{0}$; and there exists a normal positive mapping $A \rightarrow M(A)$ from $\mathscr{R}$ onto its center determined uniquely by

$$
M(A) E_{0}=E_{0} A E_{0}, \quad A \in \mathscr{R} .
$$

(iii) If $E_{1}$ is an abelian projection in $\mathscr{R}$ with central carrier 1 , then $E_{1}$ contains a vector cyclic under $\mathscr{R}$ (or $\mathfrak{A}$, if $E_{0}=E_{1}$ ) if and only if $\mathscr{R}^{\prime}$ is abelian and $\mathscr{R}^{\prime}$ (or $E_{1} \mathscr{R} E_{1}$ ) is countably decomposable.

Proof. Since $U_{g} A=U_{g} A U_{g}^{-1} U_{g}=B U_{g}$ with $B\left(=U_{g} A U_{g}^{-1}\right)$ in $\mathfrak{A}$, the elements of the form $A_{1} U_{g_{1}}+\cdots+A_{n} U_{g_{n}}$ comprise a strongoperator dense subalgebra of $\mathscr{R}$, where $A_{j}$ is in $\mathfrak{A}$ and $g_{j}$ in $G$.

As $U_{g} E_{0}=E_{0}=E_{0} U_{g}$ for $g$ in $G, E_{0}\left(A_{1} U_{g_{1}}+\cdots+A_{n} U_{g_{n}}\right) E_{0}$ $=E_{0}\left(A_{1}+\cdots+A_{n}\right) E_{0}$ so that it will suffice to prove that $E_{0} A E_{0} B E_{0}$ $=E_{0} B E_{0} A E_{0}$ for cach $A$ and $B$ in $\mathfrak{Q}$ to establish that $E_{0}$ is an abelian projection in $\mathscr{R}$.

With $\varphi=E_{0} \psi, \psi \in \mathscr{H}$, we have

$$
\left(\psi\left|E_{0} A E_{0} B E_{0}-E_{0} B E_{0} A E_{0}\right| \psi\right)=\left(\varphi\left|A E_{0} B-B E_{0} A\right| \varphi\right) \text {. }
$$

Now $E_{0}$ lies in the von Neumann algebra generated by the $U_{g}, g$ in $G$. Suppose that it lies in the strong-operator closure of the set of real linear combinations of $U_{g}$ 's, $g \in G$. For each $\varepsilon>0$, we can choose elements $g_{j} \in G$ and real constants $\lambda_{j}, j=1,2, \ldots, n$, such that

and

$$
\left\|\left(E_{0}-\sum_{j=1}^{n} \lambda_{j} U_{g_{j}}\right) B \varphi\right\|=\left\|\left(E_{0}-\sum_{j=1}^{n} \lambda_{j} U_{g g_{j}}\right) B \varphi\right\| \leqq \varepsilon / 2\left\|A^{*} \varphi\right\|
$$

$$
\left\|\left(E_{0}-\sum_{j=1}^{n} \lambda_{j} U_{g_{j}}\right) B^{*} \varphi\right\|=\left\|\left(E_{0}-\sum_{j=1}^{n} \lambda_{j} U_{g g_{j}}\right) B^{*} \varphi\right\| \leqq \varepsilon / 2\|A \varphi\|
$$

where $g$ can still be arbitrarily chosen in $G$. We then have

$$
\begin{aligned}
& \left|\left(\varphi\left|A E_{0} B-B E_{0} A\right| \varphi\right)\right| \leqq \\
& \leqq \varepsilon+\operatorname{Inf}_{g \in G}\left|\left(\varphi\left|A\left(\sum_{j=1}^{n} \lambda_{j} U_{g g_{j}}\right) B-B\left(\sum_{j=1}^{n} \lambda_{j} U_{g g_{j}}{ }^{*}\right) A\right| \varphi\right)\right|
\end{aligned}
$$

1 This fact and its connection with reduction theory was first noted in [26] i $n$ the framework of Wightman field theory. 
where the second term on the right hand side vanishes by assumption being equal to

$$
\operatorname{Inf}_{g \in G^{\prime}}\left|\left(\varphi\left|\left(A \cdot \alpha_{g}\left(B^{\prime}\right)-\alpha_{g}\left(B^{\prime}\right) A\right)\right| \varphi\right)\right| \quad \text { with } \quad B^{\prime}=\sum_{j=1}^{n} \lambda_{j} \alpha_{g_{j}}(B) .
$$

Thus $E_{0}$ is abelian, $\mathscr{R} P$ is a von Neumann algebra containing an abelian projection with central carrier the unit and is therefore of type I [12, Theorème $1 \mathrm{p}$. 123]. As $E_{0} \mathscr{R} E_{0}$ is abelian it is the center of its commutant $\mathscr{R}^{\prime} E_{0}$. Since the latter is isomorphic to $\mathscr{R}^{\prime} P$ [12, Proposition 2 p. 19], $E_{0} \mathscr{R} E_{0}$ is isomorphic with the center of $\mathscr{R}^{\prime} P$ which is obviously also the center of $\mathscr{R} P$.

It remains to prove that $E_{0}$ is approximable in the strong operator topology by sums of the form $\sum_{j=1}^{n} \lambda_{j} U_{g_{j}}$ with the $\lambda_{j}$ real. More specifically let $\mathscr{T}$ be the strong operator closure of convex linear combinations of the $U_{g_{j}}, g_{j} \in G$; and let $E_{g}, g \in G$, be the projector with range those vectors $\psi$ such that $U_{g} \psi\left(=U_{g^{-1}} \psi\right)=\psi$. The mean ergodic theorem [11; Cor. 2 and 4, p. 662] yields the fact that $E_{g}$ is the strong operator limit of $\frac{1}{n} \sum_{j=1}^{n} U_{g}^{j}$ in $\mathscr{T}$ as $n \rightarrow \infty$. If $E$ and $F$ are two projections which are the strong operator limits of operators of $\mathscr{T}$, then their intersection $E \wedge F$ is such a limit, since $E \wedge F$ is the strong operator limit of $E F E F \ldots E F$ (Method of Images) and multiplication of operators is strong operator continuous on bounded sets. Since $E_{0}=\bigwedge_{g \in G} E_{g}$ and $\underset{g \in G^{\prime}}{\wedge} E_{g}$ is strong operator limit of finite intersections of the projections $E_{g}, E_{0}$ lies in $\mathscr{T}$.

Thus (i) is proven and the specialization $P=I$ yields the first assertion of (ii). We then obtain the mapping $A \rightarrow M(A)$ by composition of the mapping $A \in \mathscr{R} \rightarrow E_{0} A E_{0} \in \mathscr{R}^{\prime} E_{0}$ with the isomorphism $T E_{0} \in \mathscr{R}^{\prime} E_{0} \leftrightarrow$ $\leftrightarrow T \in \mathscr{R}^{\prime}$, both of which are positive and normal. As the range of the first mapping is the center of $\mathscr{R}^{\prime} E_{0}$ the range of $M$ is the center of $\mathscr{R}^{\prime}$ (and $\mathscr{R}$ ). If $M_{0}$ is another mapping from $\mathscr{R}$ to $\mathscr{R}^{\prime}$ such that $M_{0}(A)$ $=E_{0} A E_{0}, \quad\left[M_{0}(A)-M(A)\right] E_{0}=0$; so that $0=\left[M_{0}(A)-M(A)\right] P$ $=M_{0}(A)-M(A)$ since $P=I$ and $M_{0}(A)-M(A) \in \mathscr{R} \wedge \mathscr{R}^{\prime}$.

Assuming now that $\psi_{0}$ is a unit vector in the range of the abelian projection $E_{1}$ such that $\mathscr{R} \psi_{0}$ is dense in $\mathscr{H}, E_{1}$ has central carrier $I$ as does the projection $E$ on the closure of $\mathscr{R}^{\prime} \psi_{0}$. Now $E \leqq E_{1}$, and abelian projections can be characterized as being minimal in the set of projections with the same central carrier $[25 ; \S 31 \mathrm{p} .332]$. Thus $E=E_{1}$ and $\psi_{0}$ is cyclic under $\mathscr{R}^{\prime}$ in $E_{1} \mathscr{H}$. Hence $I$, the projection on the closure of $\mathscr{R} \psi_{0}$ is an abelian projection in $\mathscr{R}^{\prime}[12$; Prop. 3 p. 242$]$, so that $\mathscr{R}^{\prime}$ is abelian and is the center of $\mathscr{R}\left(=\mathscr{R}^{\prime \prime}\right)$. Thus $E_{1} \mathscr{R}^{\prime} E_{1}$ is the center of the abelian algebra $E_{1} \mathscr{R} E_{1}$ and $\mathscr{R}^{\prime} E_{1}=E_{1} \mathscr{R} E_{1}$. Further $\mathscr{R}^{\prime}\left(=\mathscr{R}^{\prime} P\right)$ is isomorphic with $E_{0} \mathscr{R} E_{0}$ and is the range of the mapping $M$. 
With $\mathscr{R}^{\prime}$ abelian, we have just noted that $\mathscr{R}^{\prime} E_{1}\left(=E_{1} \mathscr{R} E_{1}\right)$ is maximal abelian on $E_{1} \mathscr{H}$. If in addition $\mathscr{R}^{\prime}\left(\right.$ or $E_{1} \mathscr{R} E_{1}$ ) is countably decomposable, $\mathscr{R}^{\prime} E_{1}$ has a vector $\psi_{1}$ cyclic for $E_{1} \mathscr{H}\left[12\right.$; Corollaire p. 20]. Since $\mathscr{R}^{\prime} \psi_{1}$ is dense in $E_{1} \mathscr{H}, \mathscr{R} \psi_{1}$ is dense in the range of an abelian projection in $\mathscr{R}^{\prime}$ with central carrier $I$. This projection must be $I$, since $\mathscr{R}^{\prime}$ is abelian.

Remark. In the foregoing proof, if (1) is valid for a single vector $\varphi \in E_{0} \mathscr{H}$ we have established that $\varphi$ is a trace vector for $E_{0} \mathscr{R} E_{0}$. If in addition $\varphi$ is cyclic under $\mathfrak{A}$, then $E_{0} \mathscr{R} E_{0}, \mathscr{R}^{\prime} E_{0}$ and $\mathscr{R}^{\prime}$ are finite von Neumann algebras.

In place of (1), the assumption that

$$
\operatorname{Inf}_{g \in G}\left|\left(\psi\left|A \alpha_{g}(B)-\alpha_{g}(B) A\right| \varphi\right)\right|=0
$$

for a single $\varphi$ in $E_{0} \mathscr{H}$ such that the closure of $\mathfrak{P} \varphi$ contains $E_{0} \mathscr{H}$, and all $\psi \in E_{0} \mathscr{H}$ would suffice to establish (i), (ii) and (iii).

Theorem 1 is especially interesting in connection with "asymptotically abelian systems" defined as follows

Definition 1. If $\{\mathfrak{A}, \alpha\}$ is a pair consisting of a $C^{*}$-algebra $\mathfrak{A}$ and $a$ homomorphism $g \rightarrow \alpha_{g}$ of the locally compact non compact group $G$ into the automorphism group of $\mathfrak{A}$ we call $\{\mathfrak{A}, \alpha\}$ an asymptotically abelian system ( $a$ weakly asymptotically abelian system) whenever, to each $\varepsilon>0$ (to each $\varepsilon>0$ and state $\Phi$ over $\mathfrak{A})$ with $A, B \in \mathfrak{U}$ there is a compact $K \subset G$ such that $g \notin K$ implies $\left\|A \alpha_{g}(B)-\alpha_{g}(B) A\right\|<\varepsilon\left(\left|\left\langle\Phi, A \alpha_{g}(B)-\alpha_{g}(B) A\right\rangle\right|<\varepsilon\right)$.

Corollary 1. Let $g \rightarrow U_{g}$ be a unitary representation of the locally compact non compact group $G$ on a Hilbert space $\mathscr{H}, \mathfrak{A}$ a $C^{*}$-algebra acting on $\mathscr{H}$ such that $\alpha_{g}(A)=U_{g} A U_{g}^{-1}$ is in $\mathfrak{A}$ for each $g$ in $G$ and let $E_{0}$ and $\mathscr{R}$ be as in Theorem 1. The conclusions (i) (ii) and (iii) of Theorem 1 are valid whenevcr $\{\mathfrak{A}, \alpha\}$ is a weakly asymptotically abelian system or whenever this is the case for $\left\{\mathfrak{I}, \alpha \mid G_{0}\right\}$, where $G_{0}$ is a non compact subgroup of $G$.

Theorem 1 is mainly useful for the study of invariant states over $C^{*}$-algebras, in the following manner:

Corollary 2. Let $\mathfrak{A}$ be a $C^{*}$-algebra, $g \rightarrow \alpha_{g}$ a homomorphism of the group $G$ into the automorphism group of $\mathfrak{A}, \Phi$ a state over $\mathfrak{A}$ invariant under $G$ i.e. such that $\Phi\left(\alpha_{g}(A)\right)=\Phi(A)$ for all $A \in \mathfrak{A}$ and $g \in G, \pi_{\Phi}$ the *-representation of $\mathfrak{A}$ on a Hilbert space $\mathscr{H}$ with cyclic vector $\Omega, U_{\Phi}$ the unitary representation of $G$ on $\mathscr{H}$ determined by

$$
\begin{cases}\left(\Omega\left|\pi_{\Phi}(A)\right| \Omega\right)=\langle\Phi, A\rangle & A \in \mathfrak{Z} \\ \pi_{\Phi}\left(\alpha_{g}(A)\right)=U_{\Phi}(g) \pi_{\Phi}(A) U_{\Phi}(g)^{-1}, & \\ U_{\Phi}(g) \Omega=\Omega & g \in G\end{cases}
$$

$\mathscr{R}$ the von Neumann algebra generated by the set $\pi_{\Phi}(\mathfrak{P}) \cup U_{\Phi}(G)$ and $E_{0}$ the orthogonal projection in $\mathscr{H}$ on the set $\left\{\psi \in \mathscr{H} \mid U_{\Phi}(g) \psi=\psi\right.$ for all $g \in G\}$. Suppose that, for all $A, B \in \mathfrak{A}$ and all vector states $\Phi$ over $\mathfrak{A}$ from $\mathscr{H}$ 
invariant under $G$

$$
\operatorname{Inf}_{g \in G}\left|\left\langle\Phi, A \cdot \alpha_{g}(B)-\alpha_{g}(B) \cdot A\right\rangle\right|=0 .
$$

Then $\mathscr{R}^{\prime}$ and $E_{0} \mathscr{R} E_{0}$ are abelian von Neumann algebras and the mapping $T \in \mathscr{R}^{\prime} \rightarrow T E_{0}$ is a ${ }^{*}$-isomorphism from $\mathscr{R}^{\prime}$ onto $E_{0} \mathscr{R} E_{0}$. Thus, for each $A \in \mathfrak{T}$ there is a unique $M_{\Phi}(A) \in \mathscr{R}^{\prime}$ such that

$$
M_{\Phi}(A) E_{0}=E_{0} \pi_{\Phi}(A) E_{0},
$$

the mapping $\pi_{\Phi}(A) \rightarrow M_{\Phi}(A) \in \mathscr{R}^{\prime}$ being linear, positive and weakoperator continuous from $\pi_{\Phi}(\mathfrak{H})$ into $\mathscr{R}^{\prime}$ and the set $\left\{M_{\Phi}(A) \mid A \in \mathfrak{A}\right\}$ being weak-operator dense in $\mathscr{R}^{\prime}$.

It is interesting that under quite general conditions, in addition to the above structure, one has the feature that $\mathscr{R}^{\prime} \cong \mathfrak{U}^{\prime \prime}$. This is the case whenever $G$ is amenable and $g \rightarrow \alpha_{g}$ is strongly continuous as will be shown in Section II below but also in other contexts as shown by

Theorem 2. $\{\mathfrak{A}, \alpha\}$ be as in Corollary 1 above with $\{\mathfrak{A}, \alpha\}$ a weakly asymptotically abelian system and $G$ locally compact, non compact and connected. Then $\mathscr{R}^{\prime}$ is contained in the weak-operator closure $\mathfrak{X}^{\prime \prime}$ of $\mathfrak{A}$ (or equivalently $\left.\mathfrak{A}^{\prime} \leqq \mathscr{R}\right)$.

Proof. From [13] some cyclic subgroup $G_{0}$ is not relatively compact; and from the definition $\left\{\mathfrak{U}, U \mid G_{0}\right\}$ is a concrete weakly asymptotically abelian system with $\Omega$ invariant under $U \mid G_{0}$ and cyclic for $\mathfrak{A}$. Now $G_{0}$ has an invariant mean (see section II below) and $\mathscr{R}_{0}$, the von Neumann algebra generated by $\mathfrak{A}$ and $U \mid G_{0}$ is contained in $\mathscr{R}$. Thus $\mathscr{R}^{\prime} \leqq \mathscr{R}_{0}^{\prime} \subseteq \mathfrak{Q}^{\prime \prime}$.

We note that if $\mathscr{R}^{\prime} \subset \mathfrak{U}^{\prime \prime}$ one then has $\mathscr{R}^{\prime}=\mathfrak{I}^{\prime \prime} \wedge \mathfrak{Q}^{\prime} \wedge U(G)$. $\mathscr{R}^{\prime}$ then consists of those elements in the center of $\mathfrak{A}^{\prime \prime}$ which commute with all $U_{g}, g \in G$. In particular the central reduction of $\mathscr{R}$ (see below) then yields mutually disjoint representations of $\mathfrak{A}$.

A further specialization of particular interest in the study of ground states at finite temperature [14] has equivalent formulations stated in the

Theorem 3. Let again $\{\mathfrak{A}, \alpha\}$ be as in Corollary 1 above with $\{\mathfrak{H}, \alpha\}$ a weakly asymptotically abelian system, $\Omega$ a vector cyclic for $\mathscr{R}$ invariant under $U_{g}$, and $\mathscr{R}^{\prime}$ contained in $\mathfrak{V}^{\prime \prime}$.

The following are equivalent

(i) $\Omega$ is cyclic for $\mathfrak{H}^{\prime}$ (or separating for $\mathfrak{Q}^{\prime \prime}$ ).

(ii) $\left\{\mathfrak{U}^{\prime} \cup U(G)\right\}^{\prime \prime}=\mathscr{R}$.

(iii) $\mathfrak{U}^{\prime \prime} \cap U(G)^{\prime} \subseteq \mathfrak{U}^{\prime \prime} \wedge \mathfrak{U}^{\prime}$.

Proof. Writing $\mathscr{R}_{1}=\left\{\mathfrak{I}^{\prime} \cup U(G)\right\}^{\prime \prime}$, (ii) reads $\mathscr{R}_{1}=\mathscr{R}$ and (iii) $\mathscr{R}^{\prime}{ }_{1} \leqq \mathfrak{U}^{\prime \prime} \cap \mathfrak{A}^{\prime}$. Since $\mathscr{R}^{\prime} \subset \mathfrak{U}^{\prime \prime}$ or $\mathfrak{U}^{\prime} \subset \mathscr{R}$ one has $\mathscr{R}_{1} \subset \mathscr{R}$. Therefore (cf. proof of Theorem 1) $E_{0} \mathscr{R}_{1} E_{0}$ is abelian and since $E_{0} \in U(G)^{\prime \prime}, E_{0} \mathscr{R}_{1} E_{0}$ is a von Neumann algebra. From (i) $E_{0} \mathscr{R}_{1} E_{0}$ is maximal abelian on $E_{0} \mathscr{H}$ so that $E_{0} \mathscr{R}_{1} E_{0}=E_{0} \mathscr{R} E_{0}$. Thus their commutants $\mathscr{R}^{\prime} E_{0}$ and $\mathscr{R}^{\prime} E_{0}$ coincide and $\mathscr{R}^{\prime}{ }_{1}=\mathscr{R}^{\prime}$ or $\mathscr{R}_{1}=\mathscr{R}$ (since $E_{0}$ has central carrier $I$ ). On the other hand (iii) trivially results from (ii) since $\mathscr{R}^{\prime}=\mathfrak{A}^{\prime \prime} \cap \mathfrak{A}^{\prime} \cap U(G)^{\prime}$ as 
we noticed above. In turn (iii) implies $\mathscr{R}_{1} \supseteqq \mathfrak{A}$ and hence that $\Omega$ is cyclic under $\mathscr{R}_{1}$. Since $\Omega$ is invariant under $U(G)$ and each $U_{g}$ induces an automorphism of $\mathfrak{A}^{\prime}, \Omega$ is then cyclic for $\mathfrak{P}^{\prime}$ i.e. one has (i).

We note that the cyclicity of $\Omega$ for $\mathfrak{Y}^{\prime}$ is a property which is conserved under the central reduction of $\mathscr{R}$ discussed below. This follows from the fact that the reduction of $\mathscr{R}$ is a reduction of $\mathscr{R}_{1}$ because of equality (ii) of the Theorem.

The extremal invariant states (E-states) over $\mathfrak{A}$. The states over $\mathfrak{A}$ invariant under $G$ (the $G$-invariant states) form a convex $w^{*}$-relatively compact subset of the topological dual $\mathfrak{A}^{*}$ of $\mathfrak{A}$. Special interest is attached to the extreme points of this convex set to which we give the name of $E$-states. $E$-states can be characterized in a variety of ways expressed in

Theorem 4. With the assumptions and notation of Corollary 2 the following are equivalent for the $G$-invariant state $\Phi$ over $\mathfrak{A}$

(i) for all $A, B \in \mathfrak{I}\left(\Omega\left|\pi_{\Phi}(A) M_{\Phi}(B)\right| \Omega\right)=\langle\Phi, A\rangle\langle\Phi, B\rangle$.

(ii) $\Omega$ is the only $G$-invariant vector of $\mathscr{H}$ i.e. $E_{0}=E_{\Omega}$ where $E_{\Omega}$ denotes the orthogonal projection in $\mathscr{H}$ on the vector $\Omega$.

(iii) For all $A_{1}, A_{2}, B \in \mathfrak{A}$

$$
\left(\Omega\left|\pi_{\Phi}\left(A_{1}\right) M_{\Phi}(B) \pi_{\Phi}\left(A_{2}\right)\right| \Omega\right)=\left\langle\Phi, A_{1} A_{2}\right\rangle\langle\Phi, B\rangle .
$$

(iv) $M_{\Phi}(A)$ is a multiple of the identity for all $A \in \mathcal{A}$.

(v) The set of operators $\pi_{\Phi}(\mathfrak{Z}) \cup U_{\Phi}(G)$ is irreducible (i.e. $\mathscr{R}$ consists of all bounded operators on $\mathscr{H}$ ).

(vi) $\mathscr{R}$ is a factor.

(vii) $\Phi$ is an extremal element of the convex set of $G$-invariant states over $\mathfrak{2}$.

Proof. We have, by the definition of $M_{\Phi}$

$$
\left(\Omega\left|\pi_{\Phi}(A) M_{\Phi}(B)\right| \Omega\right)=\left(\Omega\left|\pi_{\Phi}(A) E_{0} \pi_{\Phi}(B)\right| \Omega\right),
$$

thus since $\Omega$ is cyclic for $\pi_{\Phi}(\mathfrak{Z})$ and

$$
\langle\Phi, A\rangle\langle\Phi, B\rangle=\left(\Omega\left|\pi_{\Phi}(A)\right| \Omega\right)\left(\Omega\left|\pi_{\Phi}(B)\right| \Omega\right)
$$

(i) is equivalent to $\left.E_{0}=\mid \Omega\right)\left(\Omega \mid=E_{\Omega}\right.$. On the other hand (iii) is obtained from (i) by setting $A=A_{1} A_{2}$ and permuting $A_{2}$ with $M_{\Phi}(B) \in \mathscr{R}^{\prime}$. Conversely we obtain (i) from (iii) by setting $A_{1}=A$ and taking for $A_{2}$ an approximate identity in $\mathfrak{A}$. Now (iv) is evidently equivalent to (iii) due to the cyclicity of $\Omega$ for $\pi_{\Phi}(\mathfrak{Q})$. (iv) and (vi) are equivalent because the $M_{\Phi}(A), A \in \mathfrak{N}$, are dense in $\mathscr{R}^{\prime}$. (vi) is equivalent to (v) because $\mathscr{R}^{\prime}$ is abelian. Finally (vii) is equivalent to $(\mathrm{v})$, for, with $E^{\prime}$ a projection in $\mathscr{R}^{\prime}, \omega_{E^{\prime} \Omega} \pi_{\Phi} \leqq \Phi=\omega_{\Omega} \pi_{\Phi}$ and $\omega_{E^{\prime} \Omega} \pi_{\Phi}$ is invariant under $\alpha_{g}$. Thus $\omega_{L^{\prime} \Omega} \pi_{\Phi}=a \Phi$ and

$(\pi(A) \Omega \mid a \Omega)=\left(\pi(A) E^{\prime} \Omega \mid E^{\prime} \Omega\right)=\left(\pi(A) \Omega \mid E^{\prime} \Omega\right)$ for all $A$ in $\mathfrak{A}$.

Since $\Omega$ is cyclic under $\mathfrak{A},\left(a I-E^{\prime}\right) \Omega=0$ and, since $\Omega$ is separating 
for $\mathscr{R}^{\prime}, E^{\prime}=a I$. Hence $E^{\prime}=0$ or $I$ and $\mathscr{R}^{\prime}=\{b I\}$. Conversely, if $\mathscr{R}$ is all bounded operators and $\omega$ is an invariant linear functional such that $0<\omega<\Phi$, from [16; Lemma 2.2] $\omega=\omega_{T^{\prime} \Omega} \pi_{\Phi}$ with $T^{\prime \prime}$ a positive operator in $\mathscr{R}^{\prime}$. Since $\mathscr{R}^{\prime}$ is the scalars $\omega=a \Phi$ and $\Phi$ is extremal among the invariant states of $\mathfrak{Q}$.

Decomposition of an invariant state into E-states. With the assumptions and notation of Corollary 2, suppose, in addition, that $\mathscr{R}^{\prime}$, which we know to be abelian, is generated by its minimal projections $\left\{F_{\lambda}\right\}$ (i.e. those non-zero projections in $\mathscr{R}^{\prime}$ which dominate no other non-zero projection in $\left.\mathscr{R}^{\prime}\right)$. In this case, $\mathscr{R} F_{\lambda}$ has commutant $F_{\lambda} \mathscr{R}^{\prime} F_{\lambda}=\left\{a F_{\lambda}\right\}$ on $F_{\lambda}(\mathscr{H})$; so that $\mathscr{R} F_{\lambda}$ is the algebra of all bounded operators on $F_{\lambda}(\mathscr{H})$. The $U_{g} F_{\lambda}$ induce automorphisms of $\mathscr{R} F_{\lambda}$ and $\Omega_{\lambda}\left(=F_{\lambda} \Omega\right)$ is invariant under all $U_{g} F_{\lambda}$ and cyclic for $\pi_{\Phi}(\mathfrak{Q}) F_{\lambda}$. From Theorem $4,(\mathrm{v})$ and (vii), $\Omega_{\lambda}$ gives rise to an $E$-state $\Phi_{\lambda}$ of $\mathfrak{A}$, where $\Phi_{\lambda}(A)=\left(\pi_{\Phi}(A) \Omega_{\lambda}^{\prime} \mid \Omega_{\lambda}^{\prime}\right)$, with $\Omega_{\lambda}^{\prime}=\Omega_{\lambda} \mid\left\|\Omega_{\lambda}\right\|$ - noting that $\Omega_{\lambda} \neq 0$ since $\Omega$ is separating for $\mathscr{R}^{\prime}$ being cyclic for $\mathscr{R}$.

We have

$$
\begin{aligned}
\Phi(A)=\left(\pi_{\Phi}(A) \Omega \mid \Omega\right) & =\left(\pi_{\Phi}(A)\left(\Sigma_{\lambda} F_{\lambda}\right) \Omega \mid\left(\Sigma_{\lambda} F_{\lambda}\right) \Omega\right)=\Sigma_{\lambda}\left(\pi_{\Phi}(A) \Omega_{\lambda} \mid \Omega_{\lambda}\right) \\
& =\Sigma_{\lambda}\left\|\Omega_{\lambda}\right\|^{2}\left(\pi_{\Phi}(A) \Omega_{\lambda}^{\prime} \mid \Omega_{\lambda}^{\prime}\right)=\Sigma_{\lambda}\left\|\Omega_{\lambda}\right\|^{2} \Phi_{\lambda}(A) ; \quad(8)
\end{aligned}
$$

and $\Sigma_{\lambda}\left\|\Omega_{\lambda}\right\|^{2}=\|\Omega\|^{2}=1$. In this way we have expressed $\Phi$ as a convex sum of $E$-states $\Phi_{\lambda}$. If $\Phi=\sum_{\gamma} a_{\gamma} \Phi_{\gamma}^{\prime}$ with $\Phi_{\gamma}^{\prime}$ an $E$-state for each $\gamma$ and $a_{\gamma}>0$, then $\Phi_{\gamma}^{\prime}(A)=\left(\pi_{\Phi}(A) T^{\prime} \Omega \mid T^{\prime} \Omega\right)$ for some $T^{\prime}$ in $\mathscr{R}^{\prime}$ by $[16$, Lemma 2.2] and all $A$ in 2 . Thus $F_{\lambda} T^{\prime} \Omega=b_{\lambda} \Omega_{\lambda}^{\prime}$ and $\Phi_{\gamma}^{\prime}=\Sigma_{\lambda}\left|b_{\lambda}\right|^{2} \Phi_{\lambda}$. By extremality $\Phi_{\gamma}^{\prime}=\Phi_{\lambda_{0}}$ for some $\lambda_{0},\left|b_{\lambda_{0}}\right|=1$, and $b_{\lambda}=0$ for all $\lambda \neq \lambda_{0}$. It remains to note that if $\Sigma_{\lambda} c_{\lambda} \Phi_{\lambda}=0$, where $\Sigma\left|c_{\lambda}\right|<\infty$, then $c_{\lambda}=0$ for all $\lambda$, in order to establish the uniqueness of our decomposition of $\Phi$ into $E$-states.

For this note that $\left(\left(\pi_{\Phi}\left(A_{1}\right) U_{g_{1}}+\cdots+\pi_{\Phi}\left(A_{n}\right) U_{g_{n}}\right) \Omega_{\lambda}^{\prime} \mid \Omega_{\lambda}^{\prime}\right)$ $=\Phi_{\lambda}\left(A_{1}+\cdots+A_{n}\right)$ so that $\Sigma c_{\lambda} \omega_{\Omega^{\prime}}$ vanishes on the *-algebra generated by $\pi_{\Phi}(\mathfrak{H})$ and $\left\{U_{g}\right\}$. Since $\Sigma\left|c_{\lambda}\right|<\infty, \Sigma c_{\lambda} \omega_{\Omega^{\prime}} \lambda_{\lambda}$ is normal and vanishes on the weak-operator closure, $\mathscr{R}$, generated by this algebra. Thus $0=\Sigma c_{\lambda} \omega_{\Omega^{\prime}}\left(F_{\lambda}\right)=c_{\lambda}$ for all $\lambda$; and the uniqueness of our decomposition follows.

With suitable separability assumptions, the analogous argument using direct integral decompositions of $\mathscr{R}$ and $\mathscr{H}$ relative to $\mathscr{R}^{\prime}$, yields a (unique) direct integral decomposition of an arbitrary $G$-invariant state of $\mathfrak{A}$ as a "convex" integral of $E$-states.

\section{The Case of an Amenable Group}

We first recall a few definitions and results concerning amenable groups. For a locally compact group $G$ let us denote by $\mathscr{C}(G)$, and $\mathscr{C}_{0}(G)$, the $C^{*}$-algebras of continuous complex valued functions on $G$ respectively 
bounded, and vanishing at infinity, with the sup norm \|\|$_{\infty}$. We will sometimes denote a function $f \in \mathscr{C}(G)$ by the symbol $f(\hat{g})$ where the roofed letter indicates a dummy variable. A mean over $G$ is a positive linear form $\eta$ over the $C^{*}$-algebra $\mathscr{C}(G)$ of unit norm (i.e. $\eta(1)=1$ where 1 denotes the constant unit function). The mean $\eta$ is respectively leftor right-invariant if $\eta\{f(h \hat{g})\}=\eta\{f(\hat{g})\}$ or $\eta\{f(\hat{g} h)\}=\eta\{f(\hat{g})\}$ for all $f \in \mathscr{C}(g)$ and $h \in G$. It is (two sided) invariant if it is both left and rightinvariant. The existence of a left-invariant, right-invariant or invariant mean over $G$ are equivalent requirements which characterize a subclass of locally compact groups which we will call amenable (for the properties of amenable groups see [17]). Amenable groups can be characterized by the existence of $M$-filters as defined in [7; Definition 1]. Every abelian or solvable locally compact group is amenable. Every compact group is trivially amenable. However, no non-compact semi-simple Lie group is amenable [18] (for instance $S L(2, R)$ is not amenable). The Lorentz group is not amenable. The group of euclidean motions in 3-space is amenable since each extension of an amenable group by an amenable group is amenable [17]. If $G$ is amenable and non compact the restriction of every left- or right-invariant mean to $\mathscr{C}_{0}(G)$ vanishes. In what follows we denote by $\mathfrak{A}_{\mathscr{C}}^{G}$ the linear space of all weakly-continuous, weakly bounded functions from $G$ to $\mathfrak{A}$ (i.e. for each $\Phi$ in the topological dual $\mathfrak{A}^{*}$ of $\mathfrak{A}$ and $\left.X \in \mathfrak{A}_{\mathscr{C}}^{G},\langle\Phi, X(\hat{g})\rangle \in \mathscr{C}(G)\right)$.

We note that each $X \in \mathcal{P}_{\mathscr{C}}^{G}$ is norm-bounded (i.e. $\operatorname{Sup}_{g \in G}\|X(g)\|=\|X\|_{\infty}$ is finite) from the uniform boundedness principle [11; Chapt. II $\S 1$ p. 49]. We denote by $\mathfrak{A}^{* *}$ the von Neumann enveloping algebra of $\mathfrak{A}$ (the topological dual of $\mathfrak{2}^{*}$ with its strong topology, see [15, Chapter 12]).

In the three next paragraphs we describe means of vector or operator valued functions. Then we introduce the notion of $\eta$-asymptotic abelianness relevant to $C^{*}$-algebras acted upon by amenable groups which allows us to derive properties analogous to those of the previous section.

The mapping $M_{\eta}$ from $\mathfrak{A}$ to $\mathfrak{A}^{* *}$. This paragraph deals with averages of the functions of the type $g \rightarrow \alpha_{g}(A), A \in \mathfrak{A}$, from $G$ to $\mathfrak{A}$ with invariant means over $G$. The following lemma first gathers elementary facts on "vectorial means".

Lemma 1. Let $\mathfrak{A}$ be a $C^{*}$-algebra and $G$ a locally compact group. To each mean $\eta$ over $G$ there exists a unique mapping $\tilde{\eta}$ from $\mathfrak{A}_{\mathscr{C}}^{G}$ to $\mathfrak{A}^{* *}$ such that

$$
\langle\Phi, \tilde{\eta}(X)\rangle=\eta\{\langle\Phi, X(\hat{g})\rangle\} \text { for all } \Phi \in \mathfrak{Q}^{*} \text {. }
$$

This mapping $\tilde{\eta}$ is linear, bounded of norm not exceeding 1 (i.e. $\|\tilde{\eta}(X)\| \leqq\|X\|_{\infty}$ for all $X \in \mathfrak{Q}_{\mathscr{C}}^{G}$ ), positive (i.e. $X(g) \geqq 0$ for all $g \in G$ 8 Commun. math. Phys., Vol. 6 
implies $\tilde{\eta}(X) \geqq 0$ ) and such that, for all $A \in \mathfrak{A}$

$$
\left.\begin{array}{rl}
\tilde{\eta}(A) & =\mathrm{A} \\
\tilde{\eta}(A \cdot X) & =A \cdot \tilde{\eta}(X) \\
\tilde{\eta}(X \cdot A) & =\tilde{\eta}(X) \cdot A
\end{array}\right\}
$$

(here $A, A \cdot X$ and $X \cdot A$ are the elements of $\mathfrak{A}_{B}^{G}$ respectively defined by $A(g)=A,\{A \cdot X\}(g)=A \cdot X(g),\{X \cdot A\}(g)=X(g) \cdot A)$.

If $G$ is amenable and non compact and $\eta$ is right-invariant, $\tilde{\eta}(X)$ vanishes whenever $X$ vanishes weakly at infinity (i.e. whenever, for each $\left.\Phi \in \mathfrak{A}^{*},\langle\Phi, X(\hat{g})\rangle \in \mathscr{C}_{0}(G)\right)$. If $G$ is amenable and $\eta$ is right-invariant then $\tilde{\eta}$ is right-invariant (i.e. $\tilde{\eta}(X(\hat{g} h))=\tilde{\eta}(X(\hat{g}))$ for all $g \in G)$.

Proof. One has

$$
|\eta\{\langle\Phi, X(\hat{g})\rangle\}| \leqq \operatorname{Sup}_{g \in G^{*}}|\langle\Phi, X(g)\rangle| \leqq\|\Phi\|\|X\|_{\infty}
$$

therefore $\Phi \in \mathfrak{Q}^{*} \rightarrow \eta\{\langle\Phi, X(\hat{g})\rangle\}$ is a linear form on $\mathfrak{A}^{*}$ of norm $\leqq\|X\|_{\infty}$. For positivity, note that $\eta\{\langle\Phi, X(\hat{g})\rangle\} \geqq 0$ if $\Phi$ is a state of $\mathfrak{2}$ and $X \geqq 0$; while (12) stems form the fact that $\eta(1)=1$. For $X \in \mathfrak{A}_{6}^{G}$ and $A \in \mathfrak{U}$ one has

$$
\eta\{\langle\Phi, A \cdot X(\hat{g})\rangle\}=\eta\left\{\left\langle A^{t} \Phi, X(\hat{g})\right\rangle\right\}=\left\langle A^{t} \Phi, \tilde{\eta}(X)\right\rangle=\langle\Phi, A \tilde{\eta}(X)\rangle
$$

and analogously for right-multiplication by $A$ (we denote by $A^{t}$ the transpose of left-multiplication by $A$ and note that multiplication by $A \in \mathfrak{A}$ in $\mathfrak{A}^{* *}$ can be defined by double transposition). The last assertion of the Lemma follows immediately from the definition (11) and the fact that $\eta \mid \mathscr{C}_{0}(G)=0$. As an immediate consequence we now have

Lemma 2. Let $\mathfrak{A}$ be a $C^{*}$-algebra, $g \in G \rightarrow \alpha_{g}$ a strongly continuous homomorphism of the locally compact group $G$ into the automorphism group of $\mathfrak{A}$ (i.e. $g \in G \rightarrow \alpha_{g}(A)$ is a norm-continuous function of $g$ for all $\left.A \in \mathfrak{A}\right)$. Let $\eta$ be a mean over $G$; and let

$$
M_{\eta}(A)=\tilde{\eta}\left(\alpha_{\hat{g}}(A)\right), \quad A \in \mathfrak{A} .
$$

Then $M_{\eta}$ is a linear positive bounded mapping from $\mathfrak{A}$ to $\mathfrak{A}^{* *}$ of norm not exceeding 1 . If $G$ is amenable and $\eta$ is right invariant $M_{\eta}$ is such that

$$
M_{\eta}\left(\alpha_{g}(A)\right)=M_{\eta}(A), \quad A \in \mathfrak{A}, g \in G .
$$

Moreover the means $M_{\eta}(A), A \in \mathfrak{A}$ are $G$-invariant elements of $\mathfrak{A}^{* *}$ in the sense that, for all $g \in G$, and each left invariant mean $\eta$ over $G$,

$$
\alpha_{g}\left(M_{\eta}(A)\right)=M_{\eta}(A)
$$

where $\alpha_{g}$ denotes the action of $G$ on $2^{* *}$ obtained by double transposition.

Proof. $M$ is evidently linear, positive since $A \geqq 0$ implies $\alpha_{g}(A) \geqq 0$ for all $g \in G$, and bounded by 1 because $\left\|\alpha_{g}(A)\right\|=\|A\|, g \in G$. The invariance of $M_{\eta}$ for a right-invariant $\eta$ follows from the definition (14) 
and the last assertion of Lemma 1. For (15a) note that

for each $\Phi$ in $\mathfrak{I}^{*}$.

$$
\begin{aligned}
\left\langle\Phi, \alpha_{g}\left(M_{\eta}(A)\right)\right\rangle & =\eta\left\{\left\langle\alpha_{g}^{t}(\Phi), \alpha_{\hat{g}}(A)\right\rangle\right\}=\eta\left\{\left\langle\Phi, \alpha_{g \hat{g}}(A)\right\rangle\right\} \\
& =\eta\left\{\left\langle\Phi, \alpha_{\hat{g}}(A)\right\rangle\right\}=\left\langle\Phi, M_{\eta}(A)\right\rangle
\end{aligned}
$$

The mapping $M_{\eta}$ on the dual space $\mathfrak{I}^{*}$. In the same setting as for the last proposition we now describe the transpose of the operator $M_{\eta}$, which we again denote by $M_{\eta}$.

Lemma 3. Let $\mathfrak{A}$ be a $C^{*}$-algebra and $g \rightarrow \alpha_{g}$ a strongly continuous homomorphism of the locally compact group $G$ into the automorphism group of $\mathfrak{A}$. To each mean $\eta$ over $G$ there exists a unique mapping $M_{\eta}$ of $\mathfrak{A}^{*}$ into $\mathfrak{A}^{*}$ such that

$$
\left\langle M_{\eta}(\Phi), A\right\rangle=\left\langle\Phi, M_{\eta}(A)\right\rangle=\eta\left\{\left\langle\Phi, \alpha_{\hat{g}}(A)\right\rangle\right\}, \quad A \in \mathfrak{A}, \Phi \in \mathfrak{I}^{*} .
$$

This mapping $M_{\eta}$ is linear, positive and bounded of norm not exceeding 1. If $G$ is amenable and $\eta$ is right-invariant $M_{\eta}$ is idempotent and projects onto the set of $G$-invariant elements of $\mathfrak{Q}^{*}$ (i.e. those $\Phi \in \mathfrak{Q}^{*}$ such that $\left\langle\Phi, \alpha_{g}(A)\right\rangle$ $=\langle\Phi, A\rangle$ for all $A \in \mathcal{A}$ and $g \in G)$. If $\eta$ is an arbitrary mean and $\Phi$ is $G$-invariant $M_{\eta}(\Phi)=\Phi$.

Proof. One has, for all $\Phi \in \mathfrak{A}^{*}$ and $A \in \mathfrak{A}$

$$
\left|\left\langle\Phi, M_{\eta}(A)\right\rangle\right| \leqq\|\Phi\|\left\|M_{\eta}(A)\right\| \leqq\|\Phi\| \cdot\|A\|
$$

therefore $\Phi \rightarrow M_{\eta}(\Phi)$ is a linear mapping in $\mathfrak{Q} *$ for which $\left\|M_{\eta}(\Phi)\right\| \leqq\|\Phi\|$, $\Phi \in \mathfrak{A}^{*}$. Further, for $\Phi \geqq 0$ and $A \geqq 0$ one has $M_{\eta}(A) \geqq 0$ and $\left\langle\Phi, M_{\eta}(A)\right\rangle \geqq 0$ whence $M_{\eta}(\Phi) \geqq 0$ and $M_{\eta}$ is positive. If $\eta$ is rightinvariant $M_{\eta}(\Phi)$ is $G$-invariant for all $\Phi \in \mathfrak{Q}^{*}$ as a consequence of (16) and (15). On the other hand $G$-invariant elements of $\mathfrak{A}^{*}$ are invariant under $M_{\eta}$ for an arbitrary mean $\eta$ due to (16) and the definition of a mean. Thus for arbitrary $\Phi$ one has $M_{\eta}\left(M_{\eta}(\Phi)\right)=M_{\eta}(\Phi)$.

We note that, since $M_{\eta}$ projects onto the $G$-invariant elements of $\mathfrak{I}^{*}$, the fact that $M_{\eta}=0$ for some right-invariant mean $\eta$ implies that there does not exist any $G$-invariant state (= positive linear form of unit norm) in $\mathfrak{A}^{*}$. Conversely the absence of $G$-invariant states entails the vanishing of all operators $M_{\eta}$ (in $\mathfrak{A}^{*}$ or from $\mathfrak{A}$ to $\left.\mathfrak{A}^{* *}\right)$ for all right-invariant means $\eta$ over $G$. A last remark is that, if $\mathfrak{A}$ has a unit, the mapping $M_{\eta}$ is of unit norm (obvious by setting $A=I$ in (16)). But if $\mathfrak{A}=\mathscr{J} \oplus\{\lambda I\}$, with $\mathscr{J}$ a two-sided ideal, the $M_{\eta}$ might be trivial in the sense that $M_{\eta}(\Phi)\{A \oplus \lambda I\}=\lambda$ for all states $\Phi$ over $\mathfrak{A}$ and all $\eta$.

Means in covariant representations. We consider now as in Section I covariant representations $(\pi, U)$ of the pair $(\mathfrak{A}, G)$ and describe means of such operator valued functions as $g \rightarrow U_{g}$ or $g \rightarrow U_{g} \pi(A) U_{g}{ }^{-1}$. We begin with a lemma analogous to Lemma 1 describing means of operatorvalued functions on $G$. 
Lemma 4. Let $\mathscr{L}(\mathscr{H})$ be the algebra of bounded linear operators on a Hilbert space $\mathscr{H}, G$ a locally compact group and $\mathscr{X}$ the set of functions $g \rightarrow X(g)$ from $G$ to $\mathscr{L}(\mathscr{H})$ such that $g \rightarrow(\varphi|X(g)| \psi)$ is a continuous function on $G$ for all $\varphi, \psi \in \mathscr{H}$ and $\operatorname{Sup}\|X(g)\|=\|X\|_{\infty}$ is finite. To each mean $\eta$ over $G$ there exists a unique operator $\tilde{\eta}$ from $\mathscr{X}$ to $\mathscr{L}(\mathscr{H})$ such that

$$
(p|\tilde{\eta}(X)| \psi)=\eta\{\varphi|X(\hat{g})| \psi)\}, \quad \varphi, \psi \in \mathscr{H} .
$$

The operator $\tilde{\eta}$ is linear, bounded of norm not exceeding 1 (i.e. $\|\tilde{\eta}(X)\| \leqq$ $\leqq\|X\|_{\infty}$ for all $X \in \mathscr{X}$ ), positive (i.e. $X(g) \geqq 0$ for all $g \in G$ implies $\tilde{\eta}(X) \geqq 0$ ) and such that, for all $A \in \mathscr{L}(\mathscr{H})$, with notations analogous to those of Lemma 1

$$
\begin{aligned}
\tilde{\eta}(A) & =A \\
\tilde{\eta}(A \cdot X) & =A \cdot \tilde{\eta}(X) \\
\tilde{\eta}(X \cdot A) & =\tilde{\eta}(X) \cdot A .
\end{aligned}
$$

Furthermore $\tilde{\eta}(X), X \in \mathscr{X}$, is contained in the bicommutant of the range of $X$ (i.e. $\left.\tilde{\eta}(X) \in\{X(g) \mid g \in G\}^{\prime \prime}\right) .^{2}$

Proof. Analogous to the proof of Lemma 1. We have

$$
|\eta\{(\varphi|X(\hat{g})| \psi)\}| \leqq \operatorname{Sup}_{g \in G}|(\varphi|X(g)| \psi)| \leqq\|\varphi\|\|\psi\|\|X\|_{\infty}
$$

whence, by Riesz's Theorem, the existence of $\tilde{\eta}(X)$ of norm $\leqq\|X\|_{\infty}$ follows. Positivity and property (18) are proved as in Lemma 1 and properties (19) in an analogous manner: one has, for left multiplications

$$
\begin{aligned}
(\varphi|\tilde{\eta}(A \cdot X)| \psi) & =\eta\{(\varphi|A X(\hat{g})| \psi)\}=\eta\left\{\left(A^{*} \varphi|X(\hat{g})| \psi\right)\right\} \\
& =\left(A^{*} \varphi|\tilde{\eta}(X)| \psi\right)=(\varphi|A \tilde{\eta}(X)| \psi) .
\end{aligned}
$$

The fact that $\tilde{\eta}(X)$ is contained in the bicommutant of the range of $X$ is then an immediate consequence of (19).

Proposition 1. Let $G$ be an amenable locally compact group, $g \rightarrow U_{g} a$ strongly continuous unitary representation of $G$ on a Hilbert space, $E_{0}$ the orthogonal projector onto the $G$-invariant vectors of $\mathscr{H}$ (i.e. those $\psi \in \mathscr{H}$ such that $U_{g} \psi=\psi$ for all $\left.\psi \in \mathscr{H}\right)$. Using the notation of the preceding Lemma we then have that $\tilde{\eta}\left(U_{\hat{g}}\right)=E_{0}$ for all right or left-invariant means $\eta$ over $G$.

Proof. We first observe that, due to (19), for each $h \in G$ and a rightinvariant $\eta$

$$
\tilde{\eta}(U) U_{h}=\tilde{\eta}\left(U_{\hat{g}} U_{h}\right)=\tilde{\eta}\left(U_{\hat{g} h}\right)=\tilde{\eta}(U) .
$$

By taking means, using (19) and (18) again, it follows that

$$
\tilde{\eta}\left\{\tilde{\eta}(U) U_{h}\right\}=\tilde{\eta}(\tilde{\eta}(U))=\tilde{\eta}(U)^{2}=\tilde{\eta}(U),
$$

2 The above Lemma 1 is essentially identical with Lemma 2 from which it can be deduced by application to the universal representation of $\mathfrak{A}$. However we prefer to present straightforward independent proofs. 
thus $\tilde{\eta}(U)$ is idempotent. To prove that it is self adjoint we note that, taking adjoints in $(20), U_{h} \tilde{\eta}(U)^{*}=\tilde{\eta}(U)^{*}$, whence we derive as above

$$
\tilde{\eta}\left(U_{h} \tilde{\eta}(U)^{*}\right)=\tilde{\eta}\left(\tilde{\eta}(U)^{*}\right)=\tilde{\eta}(U) \tilde{\eta}(U)^{*}=\tilde{\eta}(U)^{*} \text {. }
$$

Hence $\tilde{\eta}(U)^{*}$ is self adjoint and $\tilde{\eta}(U)$ is a self adjoint projection such that $U_{h} \tilde{\eta}(U)=\tilde{\eta}(U) U_{h}=\tilde{\eta}(U), h \in G$, i.e. such that its range is contained in that of $E_{0}$. Thus $\tilde{\eta}(U) E_{0}=\tilde{\eta}(U)$ and, using (19),

$$
\tilde{\eta}(U) E_{0}=\tilde{\eta}\left\{U_{\hat{g}} E_{0}\right\}=\tilde{\eta}\left(E_{0}\right)=E_{0} \text {. }
$$

Therefore $\tilde{\eta}(U)=E_{0}$. Finally, for a left invariant $\eta, \eta_{1}$ given by $\eta_{1}(f(\hat{g}))=\eta\left(f\left(\hat{g}^{-1}\right)\right)$ is right invariant and $\tilde{\eta}(U)=\tilde{\eta}_{1}\left(U^{*}\right)=\tilde{\eta}_{1}(U)^{*}$ $=E_{0}^{*}=E_{0}$.

Corollary. With $G, U, \mathscr{H}$ and $\eta$ as in the preceding Proposition and $\chi a$ continuous character of $G, \tilde{\eta}(\bar{\chi} U)=E_{\chi}$, the orthogonal projection on the space of vectors $\psi \in \mathscr{H}$ such that $U_{g} \psi=\chi(g) \psi$ for all $g \in G$.

Proof. Apply the Proposition to the representation $g \rightarrow \bar{\chi}(g) U_{g}$.

Lemma 5. With $\mathfrak{A} a C^{*}$-algebra and $g \rightarrow \alpha_{g}$ a strongly continuous homomorphism of the locally compact amenable group $G$ into the automorphism group of $\mathfrak{A}$, we recall that a covariant representation $(\pi, U)$ of the system $\{\mathfrak{A}, \alpha\}$ is a pair consisting of $a^{*}$-representation $\pi$ of $\mathfrak{A}$ and $a$ strongly continuous unitary representation $U$ of $G$ such that $\pi\left(\alpha_{g}(A)\right)=U_{g} \pi(A) U_{g}{ }^{-1}$ for all $A \in \mathfrak{A}$ and $g \in G$. Using the preceding notation we then have that, for a left invariant mean $\eta$ over $G$,

$$
\tilde{\eta}\left\{U_{\hat{g}} \pi(A) U_{\hat{g}}^{-1}\right\}=\pi\left\{M_{\eta}(A)\right\} \quad\left(=m_{\eta}^{\pi}(A)\right)
$$

where the ultraweakly continuous extension of $\pi$ to $\mathfrak{A}^{* *}$ is again denoted by $\pi$. The $m_{\eta}^{\pi}(A), A \in \mathfrak{A}$, are elements of $\pi(\mathfrak{U})^{\prime \prime}$ invariant under $G$ in the sense that

$$
U_{g} m_{\eta}^{\pi}(A) U_{g}^{-1}=m_{\eta}^{\pi}(A) .
$$

Proof. Equation (21) results immediately from the comparison of definitions (16) and (17). The rest of the lemma follows from

$$
\begin{aligned}
\left(\varphi\left|U_{g} \pi\left(M_{\eta}(A)\right) U_{g}^{-1}\right| \psi\right) & =\eta\left\{\left\langle\omega_{U_{g}^{-1} \psi, U_{g}^{-1} \varphi} \pi \mid \alpha_{\hat{g}}(A)\right\rangle\right\} \\
& =\eta\left\{\left(\varphi\left|U_{g} \pi\left(\alpha_{\hat{g}}(A)\right) U_{g}-1\right| \psi\right)\right\} \\
=\eta\left\{\left(\varphi\left|\pi\left(\alpha_{g \hat{g}}(A)\right)\right| \psi\right)\right\} & =\eta\left\{\left(\varphi\left|\pi\left(\alpha_{\hat{g}}(A)\right)\right| \psi\right)\right\} \\
=\eta\left\{\left\langle\omega_{\psi, \varphi} \pi \mid \alpha_{\hat{g}}(A)\right\rangle\right\} & =\left(\varphi\left|m_{\eta}^{\pi}(A)\right| \psi\right) .
\end{aligned}
$$

Asymptotic abelianness in mean. We begin with the

Definition 2. Let $\{\mathfrak{A}, \alpha\}$ be a pair consisting of a $C^{*}$-algebra $\mathfrak{A}$ and $a$ strongly continuous homomorphism $g \rightarrow \alpha_{g}$ of the locally compact amenable group $G$ into the automorphism group of $\mathfrak{A}$. Let $\eta$ be a right or left invariant mean over $G$. The system $\{\mathfrak{A}, \alpha\}$ is called an (abstract) $\eta$-asymptotically abelian system if, for all $A, B \in \mathfrak{A}$ and each state $\Phi$ over $\mathfrak{A}$

$$
\eta\left\{\left\langle\Phi, A \cdot \alpha_{\hat{g}}(B)-\alpha_{\hat{g}}(B) \cdot A\right\rangle\right\}=0 \text {. }
$$

If $\mathfrak{A}$ is a concrete $C^{*}$-algebra acting on a Hilbert space $\mathscr{H}$ and $g \rightarrow U_{g}$ is a 
strongly continuous unitary representation of $G$ on $\mathscr{H}$ such that $\alpha_{g}(A)$ $=U_{g} A U_{g}^{-1}$ for all $A \in \mathfrak{A}, g \in G,\{\mathfrak{A}, \propto\}$ is called a concrete $\eta$-asymptotically abelian system whenever the condition above holds for all vector states $\Phi$ of $\mathfrak{A}$.

Remark 1. Each covariant representation of an abstract $\eta$-asymptotically abelian system $\{\mathfrak{U}, \alpha\}$ yields a concrete $\eta$-asymptotically abelian system.

Remark 2. For a non-compact amenable group $G$ weak asymptotic abelianness of the abstract system $\{\mathscr{A}, \alpha\}$ obviously implies $\eta$-asymptotic abelianness for all right or left invariant means $\eta$ over $G$.

Remark 3. The set of right (left) invariant means $\eta$ for which the abstract or concrete system $\{\mathfrak{U}, \alpha\}$ is $\eta$-asymptotically abelian is a $w^{*}$-closed (and thus $w^{*}$-compact) convex subset of the topological dual space of $\mathscr{C}(G)$.

Lemma 6. Let $\eta$ be a right or left invariant mean over the amenable group $G$. The concrete system $\{\mathfrak{A}, \alpha\}$ is $\eta$-asymptotically abelian if and only if $\eta\left\{U_{\hat{g}} A U_{\hat{g}}^{-1}\right\}$ (which we denote by $m_{\eta}(A)$ ) is contained in the center of the weak closure $\mathfrak{U}^{\prime \prime}$ of $\mathfrak{A}$ for all $A \in \mathfrak{A}$. Analogously the abstract system $\{\mathfrak{I}, \alpha\}$ is $\eta$-asymptotically abelian if and only if $M_{\eta}(A)$ is in the center of $\mathfrak{I}^{* *}$ for all $A \in \mathfrak{A}$.

Proof. From (19), $\tilde{\eta}\left\{\left\langle\Phi, B \alpha_{\hat{g}}(A)-\alpha_{\hat{g}}(A) B\right\rangle\right\}=\left\langle\Phi, B M_{\eta}(A)-\right.$ $\left.-M_{\eta}(A) B\right\rangle$; so that the abstract system $\{\mathfrak{A}, \alpha\}$ is $\eta$-asymptotically abelian if and only if $M_{\eta}(A)$ lies in the center of $\mathfrak{X}^{* *}$ for each $A$ in $\mathfrak{A}$. Replacing $M_{\eta}$ above by $m_{\eta}$, we conclude that the concrete system $\{\mathfrak{A}, \alpha\}$ is $\eta$-asymptotically abelian if and only if $m_{\eta}(A) \in \mathfrak{A}^{\prime} \cap \mathfrak{U}^{\prime \prime}$ for each $A \in \mathfrak{A}$.

Theorem 5. Let $\{\mathfrak{A}, \alpha\}$ be a concrete $\eta$-asymptotically abelian system acting on the Hilbert space $\mathscr{H}$ with $\eta$ a left invariant mean over the group $G$. $W e$ denote by $E_{0}$ the projector onto the subspace of vectors $\psi \in \mathscr{H}$ such that $U_{g} \psi=\psi$ for all $g \in G$ and by $\mathscr{R}$ the von Neumann algebra generated by $\mathfrak{A}$ and $U(G)$. Then

(i) $E_{0} \mathscr{R} E_{0}$ is abelian with the consequences stated in (i), (ii), (iii) of Theorem 1.

(ii) If $E_{0}$ has central carrier $I$ in $\mathscr{R}$ the mapping $m_{\eta}$ of Lemma 6 coincides on $\mathfrak{A}$ with the mapping $M$ of Theorem 1 (thus the restriction of $m_{\eta}$ to $\mathfrak{A}$ does not depend upon the choice of $\eta$ within the set considered in Remark 3 above) ${ }^{3}$.

(iii) $M$ and $m_{\eta}$ coincide also on $\mathfrak{A}^{\prime \prime}$ if and only if the conditions of Theorem 3 are realized or equivalently if the system $\left\{\mathfrak{U}^{\prime \prime}, \alpha\right\}$ is $\eta$-asymptotically abelian.

3 This explains why the $M$ could be obtained in [7] by use of an arbitrary $M$-filter (most statements and proofs in [7] are - except for the last two sections valid for amenable groups rather than only abelian groups). 
Proof. By Lemma 6 we have that $m_{\eta}(A) \cdot B-B \cdot m_{\eta}(A)=0$ for all $A, B \in \mathfrak{A}$. Now $E_{0} m_{\eta}(A) B E_{0}=E_{0} \tilde{\eta}\left(U_{\hat{g}} A U_{\hat{g}}^{-1}\right) B E_{0}=\tilde{\eta}\left(E_{0} A U_{\hat{g}}^{-1}\right) B E_{0}$ $=E_{0} A E_{0} B E_{0}$ from (19) and Proposition 1. We conclude that $E_{0} A E_{0} B E_{0}-E_{0} B E_{0} A E_{0}=0$ whence (i). In order to prove (ii) we observe that, for $A \in \mathfrak{A}$, using (19) again,

$$
m_{\eta}(A) E_{0}=E_{0} A E_{0}=M(A) E_{0} .
$$

Thus $m_{\eta}(A)=M(A)$, since $m_{\eta}(A)$ and $M(A)$ are in $\mathscr{R}^{\prime}$. To prove (iii) we first observe that Lemma 6 entails the equivalence of condition (iii) of Theorem 3, namely $\mathfrak{A}^{\prime \prime} \cap U(G)^{\prime} \subseteq \mathfrak{A}^{\prime}$, and the $\eta$-asymptotic abelianness of the system $\left\{\mathfrak{U}^{\prime \prime}, \alpha\right\}$. However the latter implies that $m_{\eta}=M$ over $\mathfrak{A}^{\prime \prime}$ using the equation above. Now this last property implies in turn property (iii) of Theorem 3 since $M\left(\mathfrak{U}^{\prime \prime}\right) \subseteq \mathscr{R}^{\prime}$ and $m_{\eta}\left(\mathfrak{U}^{\prime \prime}\right)=\mathfrak{A}^{\prime \prime} \cap U(G)^{\prime}$.

Definition 3. Let $\mathfrak{A}$ be a $C^{*}$-algebra, $G$ a locally compact amenable group, $g \rightarrow \alpha_{g}$ a strongly continuous homomorphism of $G$ into the automorphism group of $\mathfrak{A}$. A state $\Phi$ over $\mathfrak{A}$ is called $\eta$-weakly clustering whenever $\eta\left\{\left\langle\Phi, A \alpha_{\hat{g}}(B)\right\rangle\right\}=\Phi(A) \cdot \eta\left\{\left\langle\Phi, \alpha_{\hat{g}}(B)\right\rangle\right\}$ for all $A, B \in \mathfrak{A}$ with $\eta$ a right or left invariant mean over $G$. $A$ G-invariant state $\Phi$ is called weakly clustering whenever there exists a right or left invariant mean $\eta$ over $G$ for which it is $\eta$-weakly clustering i.e. such that

$$
\left\langle\Phi, A \cdot M_{\eta}(B)\right\rangle=\langle\Phi, A\rangle\langle\Phi, B\rangle
$$

Remark. For a general state $\Phi$ and denoting by the same symbol the ultraweakly continuous extension of $\Phi$ to $\mathfrak{A}^{* *}, \eta$-weakly clustering can be formulated as the requirement that $\left\langle\Phi, M_{\eta}(B)^{2}\right\rangle=\left\langle\Phi, M_{\eta}(B)\right\rangle^{2}$ for all self adjoint $B \in \mathfrak{A}$ (the last condition is known to be equivalent to $\left\langle\Phi, A \cdot M_{\eta}(B)\right\rangle=\langle\Phi, A\rangle\left\langle\Phi, M_{\eta}(B)\right\rangle$ for all $A \in \mathfrak{A}^{* *}$ [see, for example, 27, Lemma].

We can now rephrase Theorem 4 in the following way:

Theorem 4a. With $\{\mathfrak{A}, \alpha\}$ an $\eta$-asymptotically abelian abstract system and $\Phi$ an invariant state over $\mathfrak{A}$ the following are all equivalent to $(\mathrm{v}),(\mathrm{vi})$, (vii) of Theorem 4;

(i) $\Phi$ is weakly clustering.

(ii) $\Omega$ is the only $G$-invariant vector in $\mathscr{H}$.

(iii) Condition (22) holds for all right or left invariant means over $G$.

(iv) $M_{\eta}^{\pi}(A)=\langle\Phi, A\rangle \cdot I$ for all $A \in \mathfrak{A}$ and all $\eta$ such that $\{\mathfrak{A}, \alpha\}$ is $\eta$-asymptotically abelian.

\section{Examples}

\section{A. A System where the Group is compact}

Let $\mathfrak{A}$ be the full algebra of $n \times n$ complex matrices, $G$ be the compact group of unitary $n \times n$ matrices with Haar measure $\mu$ and define 
$\alpha_{U}(A)=U A U^{-1}, A \in \mathfrak{A}, U \in G$. We then have

$$
\int \alpha_{U}(A) d \mu(U)=\frac{1}{n} \operatorname{Tr}(A) \cdot I
$$

so that (cf. Lemma 6 ) the system $\{\mathfrak{U}, \alpha\}$ is $\mu$-asymptotically abelian.

Remark. If we let $H$ be the direct sum of $G$ and any locally compact, non compact group, then composing $\alpha$ with the quotient mapping modulo this group yields a system which is $\eta$-asymptotically abelian for each invariant mean $\eta$ over $H$ but not weakly asymptotically abelian.

\section{B. The Algebra of Canonical Anticommutation Relations:}

a System which is weakly but not Norm asymptotically Abelian

With $\mathfrak{A}$ the $C^{*}$-algebra of c.a.r. on $L_{2}\left(R^{n}\right)$ (generated by the smeared out bounded creation field operators $\psi(f), f$ in $L_{2}$ ), let $\alpha_{x}$ be the automorphism of $\mathfrak{A}$ determined by $\alpha_{x}(\psi(f))=\psi\left(f_{x}\right)$, $f$ in $L_{2}$ where $f_{x}(y)$ $=f(y-x)$. Then $\alpha$ is a strongly continuous representation of the translation group of $R^{n}$ by *automorphisms of $\mathfrak{A}$. We show first that the system $\{\mathfrak{Z}, \alpha\}$ is weakly asymptotically abelian.

We recall that $\mathfrak{A}$ is the linear space sum of $\mathfrak{A}_{e}$ and $\mathfrak{A}_{0}$ where $\mathfrak{A}_{e}$ is the norm closure of the linear span of monomials in $\psi(f)$ and $\psi^{*}(f)$ having an even number of terms with $\mathfrak{A}_{0}$ the same for monomials having an odd number of terms. From the commutation relations it follows that $\lim _{x \rightarrow \infty}\left\|\left[\alpha_{x}(A), B\right]_{-}\right\|=0$ for each $A$ in $\mathfrak{A}_{e}$ and all $B$ in $\mathfrak{A}$, while $\lim _{x \rightarrow \infty}\left\|\left[\alpha_{x}(A), B\right]_{+}\right\|=0$ for all $A$ and $B$ in $\mathfrak{A}_{0}$.

If we show that $\varphi\left(\alpha_{x}(A)\right) \rightarrow 0$ as $x \rightarrow \infty^{4}$ for each $A$ in $\mathfrak{A}_{0}$ and each state $\varphi$ of $\mathfrak{A}$, then $\left.\varphi\left(\left[\alpha_{x}(A), B\right)\right]_{-}\right)=\varphi_{B}\left(\alpha_{x}(A)\right)-\varphi^{B}\left(\alpha_{x}(A)\right.$, where $\varphi_{B}(C)=\varphi(C B)$ and $\varphi^{B}(C)=\varphi(B C)$, tends to 0 as $x \rightarrow \infty$ for $A$ in $\mathfrak{A}_{0}$, while $\left[\alpha_{x}(A), B\right]_{-}=\left[\alpha_{x}\left(A_{0}\right), B\right]_{-}+\left[\alpha_{x}\left(A_{e}\right) B\right]_{-}$with $A_{0}$ in $\mathfrak{A}_{0}$ and $A_{e}$ in $\mathfrak{A}_{e}$. Of course it will suffice to deal with self-adjoint $A$.

Passing to the representation associated with $\varphi$, we may assume that $\mathcal{A}$ acts on $\mathscr{H}$ with cyclic vector $\Omega$ and that $\varphi(A)=(A \Omega \mid \Omega)$. Suppose that $\alpha_{x_{n}}(A) \Omega$ tends weakly to $\psi$ in $\mathscr{H}$ where $x_{n} \rightarrow \infty$ with $n$. Then

$$
\begin{aligned}
\|\psi\|^{2} & =\lim _{n=\infty}\left(\alpha_{x_{n}}(A) \Omega \mid \lim _{m=\infty} \alpha_{x_{m}}(A) \Omega\right)=\lim _{n=\infty}\left(\lim _{m=\infty}\left(\alpha_{x_{m}}(A) \alpha_{x_{n}}(A) \Omega \mid \Omega\right)\right) \\
& =\lim _{n=\infty}\left(\lim _{m=\infty} \varphi\left(\alpha_{x_{m}}(A) \alpha_{x_{n}}(A)\right)\right. \\
& =-\lim _{n=\infty}\left(\lim _{m=\infty} \varphi\left(\alpha_{x_{n}}(A) \alpha_{x_{m}}(A)\right)=-\|\psi\|^{2} .\right.
\end{aligned}
$$

It follows that $\psi=0$ and that $\lim _{x \rightarrow \infty}\left(\alpha_{x}(A) \Omega \mid \Omega\right)=\lim _{x \rightarrow \infty} \varphi\left(\alpha_{x}(A)\right)=0$.

4 This fact follows from an argument of R. Powers (Princeton thesis to appear). We wish to thank R. Powers for helpful discussion concerning this example. 
If $\{\mathfrak{A}, \alpha\}$ was norm asymptotically abelian, $\left\|\psi\left(f_{x}\right) \psi(g)-\psi(g) \psi\left(f_{x}\right)\right\| \rightarrow 0$ as $x \rightarrow \infty$ while $\left\|\psi\left(f_{x}\right) \psi(g)+\psi(g) \psi\left(f_{x}\right)\right\|=0$ for all $x$; so that $\left\|\psi\left(f_{x}\right) \psi(g)\right\| \rightarrow 0$ as $x \rightarrow \infty$. If $f_{x}$ and $g$ have compact disjoint supports, however, $\left\|\psi\left(f_{x}\right) \psi(g)\right\|=\|f\|\|g\| \neq 0$ if both $f$ and $g$ are non zero. Thus $\{\mathfrak{A}, \alpha\}$ is not norm asymptotically abelian.

In the last examples all $M_{\eta}$ are zero, so that there are no $G$-invariant states (cf. the remark following Lemma 3).

\section{The C*-Algebra of a locally compact Abelian Group Acted upon by the dual Group}

With $G$ a locally compact non discrete abelian group and $\hat{G}$ its non compact dual group topologized as usual, the $C^{*}$-algebra of $G$ is the algebra $\mathscr{C}_{0}(\hat{G})$ of continuous functions on $\hat{G}$ vanishing at infinity with pointwise multiplication, complex conjugation and the Sup norm \|\|$_{\infty}$. For each $k \in \hat{G}$ we define $\left\{\alpha_{k}(f)\right\}(p)=f(p-k), f \in \mathscr{C}_{0}(\hat{G}), p \in \hat{G}$. Since $\mathscr{C}_{0}(\hat{G})=\mathfrak{A}$ is abelian we have a trivially asymptotically abelian system $\{\mathfrak{A}, \alpha\}$. Now the set $A(\hat{G})$ of Fourier transforms of $L_{1}$-functions on $G$ is a dense sub-*algebra of $\mathfrak{A}$ and therefore, to prove that all means on $\mathfrak{A}$ and on $2^{*}$ vanish, it suffices to verify that they vanish on $A(\hat{G})$. Now for $f \in A(\hat{G})$ and $\mu$ a bounded measure over $\hat{G}$ one has

$$
\left\langle\mu, M_{\eta}(f)\right\rangle\left(=\left\langle M_{\eta}(\mu), f\right\rangle\right)=\eta\left\{\left\langle\mu \alpha_{\hat{k}}(f)\right\rangle\right\}=0 ;
$$

since $\left\langle\mu, \alpha_{\hat{k}}(f)\right\rangle(=f * \mu)$ is an element of $A(\hat{G}) \subset \mathscr{C}_{0}(\hat{G})$.

\section{The Twisted Convolution Algebra $\overline{L_{1}(E, \sigma)}$}

Acted upon by the Translations of the underlying Symplectic Space $E$

Let $E$ be a finite-dimensional real vector space equipped with a nondegenerate bilinear form $\sigma$. The twisted convolution algebra $L_{1}(E, \sigma)$ over $E$ (see [19]) is obtained by taking the set of Lebesgue-integrable functions over $E$ with its $L_{1}$-norm \|\|$_{1}$ and the following *-operation and product $\times$ :

$$
\left\{\begin{aligned}
f^{*}(\psi) & =\overline{f(-\psi)}, & & f, g \in L_{1}(E, \sigma) \\
(f \times g)(\psi) & =\int e^{-i \sigma(\xi, \psi)} f(\xi) g(\psi-\xi) d \xi, & & \psi \in E
\end{aligned}\right.
$$

$L_{1}(E, \sigma)$ is shown to be a Banach *-algebra possessing a unique irreducible representation up to unitary equivalence. Since this representation is faithful, the completion $\overline{L_{1}(E, \sigma)}=\mathfrak{A}$ of $L_{1}(E, \sigma)$ in the operator norm is a $C^{*}$-algebra which is shown to be isomorphic to the $C^{*}$-algebra of compact operators on the irreducible representation space. Furthermore the *-operation and product $(23)$ can be extended to the set $M_{1}(E, \sigma)$ of bounded complex measures over $E$ by defining, for each continuous function $f$ on $E$ with compact support

$$
\begin{cases}\left\langle\mu^{*}, f\right\rangle=\overline{\left\langle\mu, f^{*}\right\rangle}, & \mu, v \in M_{1}(E, \sigma) \\ \langle\mu \times v, f\rangle=\int d \mu(\eta) \int d \nu(\xi) e^{-i \sigma(\eta, \xi)} f(\xi+\eta) & \end{cases}
$$


$M_{1}(E, \sigma)$ with the \|\|$_{1}$-norm of measures thus becomes a $*$-Banach algebra in which $L_{1}(E, \sigma)$, identified with the set of measures absolutely continuous with respect to Lebesgue-measure, is a (two-sided) *-ideal. Furthermore the definitions (24) are compatible with the extension of the operation $(23)$ on $L_{1}(E, \sigma)$ to its $C^{*}$-completion $\mathfrak{A}$.

We now assign to each $u \in E$ the ${ }^{*}$-automorphism $\alpha_{u}$ of $\mathfrak{U}$ defined by

$$
\alpha_{u}(a)=\delta_{u} \times a \times \delta_{-u} \quad a \in \mathfrak{A}
$$

where $\delta_{u}$ is the Dirac measure on $E$ at $u$. Since, according to (24), one has

$$
\left\{\begin{array}{l}
\delta_{u}^{*}=\delta_{-u}=\delta_{u}^{-1} \\
\delta_{u} \times \delta_{v}=e^{i \sigma(u, v)} \delta_{u+v}
\end{array}\right.
$$

$u \rightarrow \alpha_{u}$ is a homomorphic mapping of the additive group of $E$ into the group of $*$-automorphism of $\mathfrak{A}$. Further, one easily calculates from (24) that, for $f \in L_{1}(E, \sigma)$,

$$
\left\{\alpha_{u}(f)\right\}(\psi)=e^{-2 i \sigma(u, \psi)} f(\psi) \text { a.e. in } \quad \psi \in E .
$$

We now show that

for each $a \in \mathfrak{A}$ and each continuous linear form $\Phi$ over $\mathfrak{A}$ one has

and

$$
\left\langle\Phi, \alpha_{u}(a)\right\rangle \rightarrow\langle\Phi, a\rangle \text { as } u \rightarrow 0
$$

that is,

$$
\left\langle\Phi, \alpha_{u}(a)\right\rangle \rightarrow 0 \quad \text { as } \quad u \rightarrow \infty
$$

$$
\left\langle\Phi, \alpha_{\hat{u}}(a)\right\rangle \in \mathscr{C}_{0}(E)
$$

Since each continuous linear form on $\mathfrak{A}$ is the difference of two positive forms and since $L_{1}(E, \sigma)$ is dense in $\mathfrak{A}$ it suffices to prove (28) and (29) for a positive form $\Phi$ and for $a=f, f \in L_{1}(E, \sigma)$. Now each positive form $\Phi$ on $L_{1}(E, \sigma)$ corresponds to a function $\varphi \in L_{\infty}(E)$; so that, by (27),

where $\widehat{f \varphi}$ is the Fourier-transform of the $L_{1}$-function $f \varphi$.

$$
\left\langle\Phi, \alpha_{u}(f)\right\rangle=\int e^{-2 i \sigma(u, \psi)} f(\psi) \varphi(\psi) d \psi=\widehat{f \varphi}(\sigma u)
$$

As a consequence of the weak continuity of the mapping $u \in E \rightarrow$ $\rightarrow \alpha_{u}(a) \in \mathfrak{A}$ expressed by (28) it is known that this mapping is also continuous in the norm topology of $\mathfrak{A}[28 ; 10.2$. Corollary]. On the other hand it follows immediately from (28) that one has the following asymptotic abelian property:

$$
\left\langle\Phi, \alpha_{u}(a) \cdot b-b \cdot \alpha_{u}(a)\right\rangle \rightarrow 0, \text { as } u \rightarrow \infty, \begin{aligned}
& a, b \in \mathfrak{A} \\
& \Phi \in \mathfrak{A}^{*}
\end{aligned}
$$

and that, for every mean $\eta$ on $E$ and all $a \in \mathfrak{U}$ and $\Phi \in \mathfrak{A}^{*}$

$$
\eta\left(\left\langle\Phi, \alpha_{\hat{u}}(a)\right\rangle\right)=\left\langle\Phi, M_{\eta}(a)\right\rangle=\left\langle M_{\eta}(\Phi), a\right\rangle=0 .
$$

Therefore all means on $\mathfrak{A}$ (or on $\mathfrak{A}^{*}$ ) vanish. 
Acknowledgements. Our thanks are due to G. Gallavotti, D. Ruelle and A. Verbeure for enlightening discussions and to R. HaAg, N. Hugenholtz and M. WINNINK for communicating to us an early version of their work. Grants from the Ministère de l'Education Nationale and from ONR and NSF made possible the collaboration during June and July 1966 when these results were obtained. We wish to express our gratitude to Dr. L. Mochtane for the kind hospitality of the I.H.E.S. and to the University of Pennsylvania for the use of its facilities during the preparation of this paper.

\section{References}

1. HAAG, R., and D. KASTLER: An algebraic approach to quantum field theory. J. Math. Phys. 5, 848 (1964).

2. Doplicher, S.: An algebraic spectrum condition. Commun. Math. Phys. 1, 1 (1965).

3. HAAG, R.: Mathematical structure of the BCS model. Nuovo Cimento 25, 281 (1962).

4. Araki, H., and E. J. Woods: Representations of the canonical commutation relations describing a nonrelativistic infinite free Bose gas. J. Math. Phys. 4, 637 (1963).

4a. Robinson, D. W.: The ground state of the Bose gas. Commun. Math. Phys. 1, 159 (1965).

5. Doplicher, S., D. Kastler, and D. W. Robinson: Covariance algebras in field theory and statistical mechanics. Commun. Math. Phys. 3, 1 (1966).

6. Ruelle, D.: States of physical systems. Commun. Math. Phys. 3, 133 (1966).

7. Kastler, D., and D. W. Robinson: Invariant states in statistical mechanics. Commun. Math. Phys. 3, 151 (1966).

8. Robinson, D. W., and D. Ruelle: Extremal invariant states. I.H.E.S. Ann. Inst. H. Poincaré, to appear.

9. LANFORD, O., and D. Ruelle: Integral representations of invariant states on $B^{*}$-algebras. J. Math. Phys., to appear.

9a. Størmer, E.: Large groups of automorphisms of $C^{*}$-algebras. Commun. Math. Phys. 5, 1 (1967).

10. Ruelle, D.: The states of classical statistical mechanics, Preprint 1966.

11. Dunford, N., and J. T. Schwartz: Linear operators, Part I, New York: Interscience Publ. 1958.

12. Dixmier, J.: Les algèbres d'opérateurs dans l'espace hilbertien. Paris: GauthierVillars, 1967.

13. Kadison, R. V.: A topological Burnside theorem, to appear.

14. HaAg, R., N. Hugenholtz, and M. Winnink: On the equilibrium states in quantum statistical mechanics. Preprint 1966.

15. Drxmier, J.: Les $C^{*}$-algèbres et leurs représentations. Paris: Gauthier-Villars 1964.

16. Dye, H. A.: The Radon Nicodym theorem for finite rings of operators. Trans. Am. Math. Soc. 72, 243 (1952).

17. Pier, J. P.: Sur une classe de groupes localement compacts remarquables du point de vue de l'Analyse harmonique. Thèse de 3ème Cycle, Nancy (1965).

18. Takenouchi, O.: Sur une classe de fonctions continues de type positif sur un groupe localement compact. Math. J. Okayama Univ. 4, 143 (1955)

19. Kastler, D.: The $C^{*}$-algebras of a free Boson field. Commun. math. Phys. 1, 14 (1965).

20. Følner, E.: On groups with full Banach mean value. Math. Scand. 3, 243-254 (1955). 
21. Ulanicki, A.: Amenable topological groups. Univ. of Seattle (1965).

22. Reiter, H.: On some properties of locally compact groups. Univ. of Utrecht (1965).

23. - Sur la propriété $\left(P_{1}\right)$ et les fonctions de type positif. C.R. Acad. Sci. Paris, 258 (1964); pp. 5, 134-5, 135.

24. - The convex hull of translates of a function in $L^{1}, L$. London Math. Soc. $\mathbf{3 5}$, $5,(1960)$.

25. Kadison, R. V.: Unitary invariants for representations of operator algebras. Ann. Math. 66, 304 (1957).

26. Reen, H., and S. Schlieder: Ưber den Zerfall der Feldoperatoralgebra im Falle einer Vakuumentartung. Nuovo Cimento 26, 32 (1962).

27. Kadison, R. V.: The trace in finite operator algebras. Proc. Am. Math. Soc. 12, 973 (1961).

28. Hille, E., and R. S. Philltips: Functional analysis and semi groups. Am. Math. Soc. Coll. Pub. Providence R.I. (1957). 